cells aligned parallel to the rotation axis and at a tangent to the inner core. Thus, a prediction from the strong cooling above the central Asian cyclone in the calculations is that there will be greater solidification rates in a broad equatorial region beneath Malaysia, exactly where seismologists locate the eastern hemispheric peak in seismic velocity. Moreover, laboratory experiments on solidifying ingots ${ }^{7}$ show that rapid solidification gives a crystal fabric - less oriented, finer grained - that Aubert et al. ${ }^{4}$ argue is also consistent with the greater seismic isotropy and attenuation observed in the eastern hemisphere.

Case proved? Perhaps - although I find that it takes an eye of faith to focus on the similarities beneath central Asia and overlook dissimilarities, such as the strong anticyclone and flux patch south of Africa that are evident in the Earth but not in Aubert and colleagues' model. And it is an interesting question whether the variations with solidification rate in small-scale laboratory experiments ${ }^{7}$ can be scaled up and slowed down to the conditions of inner-core growth.
Aubert et al. ${ }^{4}$ estimate that, to solidify a 100 -km-thick layer with seismically observable east-west differences, the pattern of solidification rates must be maintained for at least 100 million to 300 million years - and this is for a young and rapidly growing inner core. Over this period, the pattern of mantle cooling must be maintained, and the inner core held almost fixed relative to the mantle by gravitational coupling (allowing small torsional oscillations about equilibrium). The timing is tight, but seems feasible.

A fascinating implication of the picture provided by Aubert et al. ${ }^{4}$ is that major differences in the configurations of the plates and the pattern of mantle convection in the past could have been imprinted on the inner core at earlier stages of its growth. Perhaps the inner-core structure is something like an onion, with different layers recording different mantle epochs. Could seismologists 'peel the onion' to reveal evidence of the history of both inner-core growth and mantle convection? Newly published images of the inner core reveal a seismically distinct 'inner inner core' of radius $600 \mathrm{~km}$, outside which the eastern hemisphere, but not the western, is layered ${ }^{8}$. The calculations of Aubert et al. offer a new way to interpret such images, and give insight into this most central of problems in Earth's evolution.

John Lister is at the Institute of Theoretical

Geophysics, Department of Applied

Mathematics and Theoretical Physics, University

of Cambridge, Cambridge CB3 OWA, UK.

e-mail: lister@damtp.cam.ac.uk

1. Tanaka, S. \& Hamaguchi, H. J. Geophys. Res. 102, 2925-2938 (1997).

2. Creager, K. C. J. Geophys. Res. 104, 23127-23139 (1999).

3. Amit, H. \& Olson, P. Phys. Earth Planet. Inter. 155, 120-139 (2006).

4. Aubert, J., Amit, H., Hulot, G. \& Olson, P. Nature 454, 758-761 (2008)

5. Bloxham, J. \& Jackson, A. Rev. Geophys. 29, 97-120 (1991)

6. Masters, G. et al. in Earth's Deep Interior Vol. 117 (eds Karato, S. et al.) 63-87 (Am. Geophys. Union, 2000)

7. Bergman, M. I., Agrawal, S., Carter, M. \& MacleodSilberstein, M. J. Crystal Growth 255, 204-211 (2003).

8. Sun, X.\& Song, X. Earth Planet. Sci. Lett. 269, 56-65 (2008).

9. Gonnermann, H. M., Jellinek, A. M., Richards, M. A. \& Manga, M. Earth Planet. Sci. Lett. 226, 53-67 (2004).

\title{
TUBERCULOSIS
}

\section{Shrewd survival strategy}

\author{
Steven A. Porcelli
}

\section{Mycobacterium tuberculosis modulates its virulence to cause persistent but often subclinical infection. This strategy is regulated in part by a feedback loop that controls the secretion of a small subset of bacterial proteins.}

The bacterium that causes tuberculosis is one of the most successful pathogens. Its spread among humans has been so efficient that as much as one-third of the world's population is now believed to be infected ${ }^{1}$. In most cases, these infections cause clinically silent disease, which is likely to remain permanently dormant unless the host's immunity is substantially compromised. The extraordinary stealth and opportunism that Mycobacterium tuberculosis exhibits results from the complex and delicately coordinated way in which it interacts with its host - a process controlled in part by a specialized bacterial protein-secretion system called ESX-1. On page 717 of this issue, Raghavan et al. ${ }^{2}$ provide insight into how mycobacteria

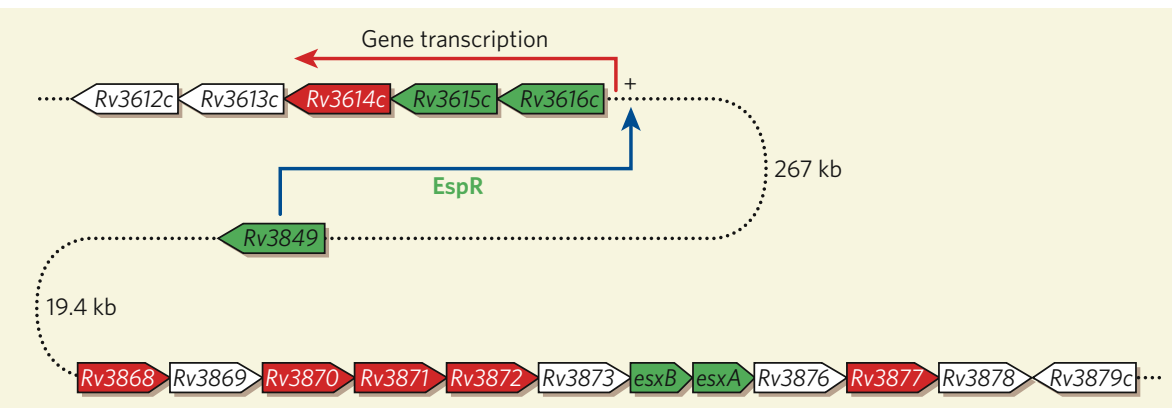

Figure 1 Genes involved in ESX-1 secretion. At least three separate regions of the M. tuberculosis chromosome encode genes that are involved in the ESX-1-secretion system. The es $x B A$ operon encodes the main secreted products ESAT- 6 and CFP-10. Raghavan et al. ${ }^{2}$ identify the protein product of the $R v 3849$ gene - which is some distance (19.4 kilobases) away from the es $x B A$ operon - as the EspR protein. This protein is both a secreted substrate of ESX-1 and a positive transcriptional regulator of a third region, the $R v 3616 c-R v 3612 c$ operon, which is also involved in ESX-1-mediated virulence. Genes encoding known secreted substrates of ESX-1 are shown in green, and those encoding structural or functional components of the ESX-1 secretion apparatus are in red. Genes in white are of unknown function, although they are presumed to be somehow involved in the ESX-1 system.

might maintain ESX-1 activity at just the right level, perhaps allowing these pathogens to avoid excessive virulence so that they can patiently pursue their low-key yet incredibly effective strategy for long-term survival.

Mycobacteria lack the specialized type I-VI secretion systems that have been well characterized in Gram-negative bacteria. Instead, their virulence is mediated in part by ESX-1, which is also found in other Gram-positive bacteria. Two proteins secreted by the ESX-1 system, ESAT-6 and CFP-10, are prominent targets of the immune system in animals, including humans, infected with M. tuberculosis ${ }^{3}$. These proteins are encoded together as part of a coordinated unit of genetic material, the esxBA operon. Genomic studies ${ }^{4}$ predicted that genes surrounding es $x B A$ are likely to encode components of a secretory apparatus responsible for the export of ESAT-6 and CFP10 out of the bacterial cytoplasm. This prediction was soon validated ${ }^{5,6}$, defining ESX-1 as a prototypical virulence-associated mycobacterial protein-secretion system.

Exactly how ESX-1 contributes to mycobacterial virulence remains a puzzle, although various observations have led to several hypotheses that are not mutually exclusive. For example, ESX-1 mutants grow poorly during the early phase of infection in mice and in macrophages ${ }^{6,7}$ (immune cells that engulf pathogens by phagocytosis). Moreover, in infected dendritic cells (another type of phagocytic immune cell), ESX-1 helps M. tuberculosis escape from phagosome vesicles - in which they are captured for destruction - into the cytoplasm, where they can replicate ${ }^{8}$. Because purified ESAT- 6 acts as a membrane-disrupting toxin in vitro ${ }^{9}$, this protein could directly mediate phagosomal escape. However, ESX-1 also 
hinders phagosome maturation in macrophages through a process that does not require ESAT- 6 or other known substrates secreted by this system $^{10}$. Taken together, these data indicate that ESX-1 modulates innate immune responses of the infected host through several mechanisms, which probably involve ESX-1-mediated secretion of ESAT- 6 and other unidentified factors.

Raghavan and colleagues ${ }^{2}$ identify a previously unknown component of ESX-1 that is not only a central regulator of it but is also secreted by this system. They find that, in M. tuberculosis, the Rv3849 gene - which is located some distance from the main ESX-1 gene cluster surrounding the es $x B A$ operon - is required for ESX-1 function. The protein product of Rv3849, EspR, is highly similar to a gene transcription factor of the harmless soil bacterium Bacillus subtilis. The authors find that EspR is also a DNA-binding transcriptional regulator $-R v 3849$ deactivation leads to changes in the transcription of a few operons in the $M$. tuberculosis genome, including the $R v 3616 c-3612 c$ cluster, which encodes at least two ESX-1-secreted proteins and is required for the functioning of ESX-1 ${ }^{11}$ (Fig. 1).

Although it is not surprising that mycobacteria express a transcription factor that regulates the expression of ESX-1 components, that EspR is itself secreted by ESX-1 is an unexpected result. The authors propose that secretion of this protein constitutes an unusual feedback loop that could be part of a finely tuned control process to prevent excessive or prolonged activity of ESX-1 during infection of mammalian cells. Although secretion of a regulatory protein as a mechanism for diminishing its activity inside the cell has been described before, EspR might represent the first example of a transcription factor that is actively exported from the cell by the same secretion system that it induces.

One can imagine how EspR could impose a limit on the level and duration of ESX-1 activity. When initially expressed, this protein could be essential for activating ESX-1 to secrete high levels of virulence-promoting proteins, thus allowing $M$. tuberculosis to establish its infection in the host. Once infection is achieved, secretion of EspR might lead to a reduction of its transcriptional activity within the bacterium and diminished ESX-1 activity. This would partially attenuate virulence, thus favouring either bacterial persistence or a slow, chronic infection in order to enhance transmission.

Can EspR production be turned on or off, and - if so - what external stimuli and bacterial sensing and signalling molecules could be responsible for this? Are other components of the ESX-1 system separately regulated by factors distinct from EspR? These questions, together with the identification of other secreted ESX-1 substrates and their mechanisms of action in mammalian host cells, should provide many opportunities for deciphering the unique logic of mycobacterial virulence strategies.

It will also be interesting to determine whether EspR has any specific function once it has been exported from the bacterial cell. Because at least two other ESX-1 secreted products - ESAT- 6 and CFP-10 - are major targets for the immune response, it is possible that EspR is also a prominent mycobacterial antigen. If so, this could have implications for the development of new vaccines and diagnostic tools for tuberculosis.

Steven A. Porcelli is in the Departments of Microbiology and Immunology, and of Medicine, Albert Einstein College of Medicine, 1300 Morris Park Avenue, Bronx, New York 10461, USA. e-mail: porcelli@aecom.yu.edu
1. Dye, C., Scheele, S., Dolin, P., Pathania, V. \& Raviglione, M. C. J. Am. Med. Assoc. 282, 677-686 (1999).

2. Raghavan, S., Manzanillo, P., Chan, K., Dovey, C. \& Cox, J. S. Nature 454, 717-721 (2008).

3. Sorensen, A. L., Nagai, S., Houen, G., Andersen, P. \& Andersen, A. B. Infect. Immun. 63, 1710-1717 (1995).

4. Gey van Pittius, N. C. et al. Genome Biol. doi:10.1186/ gb-2001-2-10-research0044 (2001).

5. Converse, S. E. \& Cox, J. S. J. Bacteriol. 187, 1238-1245 (2005).

6. DiGiuseppe Champion, P. A. \& Cox, J. S. Cell Microbiol. 9, 1376-1384 (2007).

7. Stanley, S. A., Raghavan, S., Hwang, W. W. \& Cox, J. S Proc. Natl Acad. Sci. USA 100, 13001-13006 (2003).

8. van der Wel, N. et al. Cell 129, 1287-1298 (2007).

9. Hsu, T. et al. Proc. Natl Acad. Sci. USA 100, 12420-12425 (2003).

10. MacGurn, J. A. \& Cox, J. S. Infect. Immun. 75, 2668-2678 (2007).

11. MacGurn, J. A., Raghavan, S., Stanley, S. A. \& Cox, J. S Mol. Microbiol. 57, 1653-1663 (2005).

\section{OPTICS}

\section{Electronic eyeballs}

\section{Takao Someya}

\section{The ability to fabricate silicon optoelectronic devices on a curved surface will lead to imaging systems with exceptional characteristics. This innovative technology will find diverse applications.}

All animals have curved imagers for their eyes. By contrast, artificial vision systems such as digital and video cameras have to rely on flat image-recording surfaces. These artificial imagers are made with silicon microfabrication technologies to produce the necessary network of semiconductor photodetectors, and they can now create pictures with more than ten million pixels. But there remains the big problem of producing bright, distortion-free images with a flat imager. Given the distortion that occurs at the edges of lenses, multiple combinations of different lenses are required for effective imaging. Consequently, lens arrangements are heavy, expensive and produce darker results than they would otherwise do ${ }^{1}$.

On page 748 of this issue, Rogers and colleagues (Ko et al. $)^{2}$ describe how they have drawn inspiration from animals' eyes and have succeeded in eliminating these fundamental limitations of conventional artificial-vision systems. Their electronic eye camera (Fig. 1) is based on silicon electronics that is designed to have full mechanical compressibility-stretchability, meaning that it can be moulded onto a hemispherical substrate.

The authors' method is outlined in Figure 1 of their paper (page 749). It depends on two main advances. The first is the fabrication, on a silicon wafer, of a network of semiconductor photodetectors that can tolerate elastic compressibility despite being subjected to high levels of strain (typically exceeding 50\%). The crucial features that allow such compressibility are the thin metallic wires that interconnect the photodetectors. The second innovation is the use of elastomeric elements that can transform a photodetector network initially made in a planar configuration into hemispherical

\begin{abstract}
Figure 1 | The electronic eye camera. This device shows the integration of the concave photodetector system devised by Ko et al. ${ }^{2}$ into a miniature camera that has a single, simple lens. Apart from the lens at the top, the hemispherical cap would not normally be transparent. The camera is about 2 centimetres in diameter. (Photo courtesy of J. A. Rogers.)
\end{abstract}

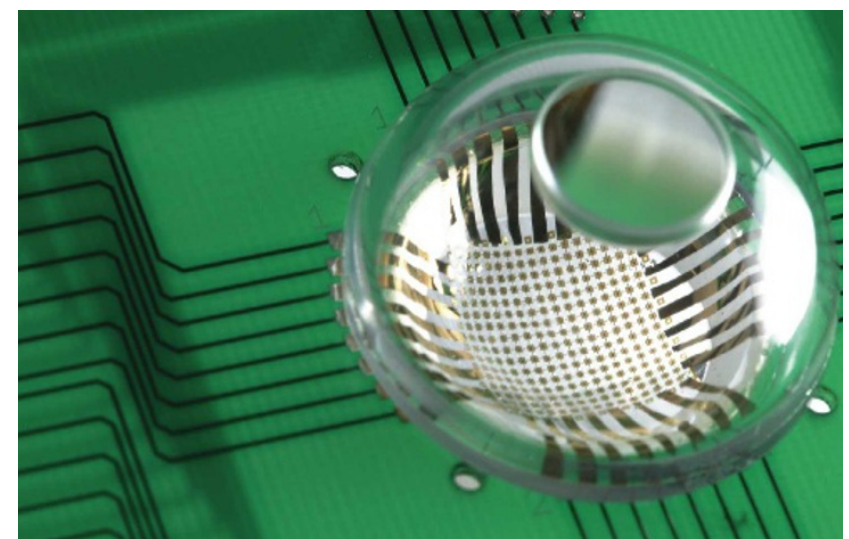

